\title{
WHAT IS THE BEST PERFUSION TEMPERATURE FOR CORONARY REVASCULARIZATION?
}

Richard M. Engelman, MD ${ }^{\mathrm{a}, \mathrm{d}, \mathrm{e}}$ A. Bernard Pleet, MD ${ }^{\mathrm{b}, \mathrm{e}}$ John A. Rousou, $\mathrm{MD}^{\mathrm{a}, \mathrm{d}, \mathrm{e}}$ Joseph E. Flack, III, MD ${ }^{\mathrm{a}, \mathrm{d}}$ David W. Deaton, MD ${ }^{\mathrm{a}, \mathrm{d}}$ Cheryl A. Gregory, $\mathrm{RN}^{\mathrm{a}}$ Penelope S. Pekow, $\mathrm{PhD}^{\mathrm{c}}$
Background: A National Institutes of Health-funded clinical trial of patients undergoing coronary artery bypass randomized perfusate and myocardial preservation to cold, tepid, or warm temperatures. The goal of the trial was to evaluate neurologic function before and after operation (4 days and 1 month after operation) and to measure hematologic data for fibrinolytic potential. Methods: The three groups comprised 116 patients who completed neurologic evaluation by means of the Mathew scale out of 130 entered into the trial (37 cold group, 50 tepid, and 43 warm). Twenty-five patients had complete hematologic studies done. All three groups were comparable before operation. The myocardial preservation protocol used blood cardioplegic solution at cold $\left(8^{\circ}\right.$ to $\left.10^{\circ} \mathrm{C}\right)$, tepid $\left(32^{\circ} \mathrm{C}\right)$, or warm $\left(37^{\circ} \mathrm{C}\right)$ temperature and the systemic perfusate temperature during cardiopulmonary bypass was $20^{\circ}$ (cold), $32^{\circ} \mathrm{C}$ (tepid), or $37^{\circ} \mathrm{C}$ (warm). Results: Patients in the cold group had a longer duration of intubation and postoperative hospitalization and a slightly but significantly higher peak postoperative creatine kinase MB level than patients in the warm group. There were no deaths. There was deterioration in Mathew scale findings in all three groups, and no distinction could be made between groups. However, a significantly higher number in the cold group had an abnormal postoperative neurologic examination result that prompted computed tomographic scanning (18.9\% cold, $2 \%$ tepid, $9.3 \%$ warm). A cerebrovascular accident was documented by computed tomographic scanning in $8.1 \%, 0 \%$, and $4.7 \%$ of patients in the cold, tepid, and warm groups, respectively (not significant). Hematologic data documented significantly increased fibrinolytic potential in the warm group. Conclusions: Perfusion temperature is a factor in recovery from cardiopulmonary bypass. Cold has more adverse neurologic sequelae that prompt computed tomographic scanning whereas warm has more activation of fibrinolytic potential. Tepid is the best temperature for optimizing recovery from cardiopulmonary bypass. (J Thorac Cardiovasc Surg 1996;112:1622-33)
From the Departments of Surgery ${ }^{\mathrm{a}}$ and Neurology, ${ }^{\mathrm{b}}$ Baystate Medical Center, Springfield, Mass.; the Department of Biostastistics and Epidemiology, ${ }^{\mathrm{c}}$ School of Public Health and Health Sciences, University of Massachusetts, Amherst, Mass.; University of Connecticut School of Medicine, ${ }^{d}$ Farmington, Conn.; and Tufts University School of Medicine, ${ }^{\mathrm{e}}$ Boston, Mass.

Supported by National Institutes of Health grant No. 1R01 HL 48631-02.

Read at the Seventy-sixth Annual Meeting of The American Association for Thoracic Surgery, San Diego, Calif., April 28-May 1, 1996.

Received for publication May 6, 1996; revisions requested June 18, 1996; revisions received July 12, 1996; accepted for publication July 15, 1996.

Address for reprints: Richard M. Engelman, MD, Baystate Medical Center, 759 Chestnut St., Springfield, MA 01107.

Copyright (C) 1996 by Mosby-Year Book, Inc.

$0022-5223 / 96 \$ 5.00+0 \quad \mathbf{1 2 / 6 / 7 6 5 1 0}$
The concept of warm heart operation was initiated at the University of Toronto in 1987.' Before 1987, cold preservation had been the gold standard and cold perfusion during cardiopulmonary bypass (CPB) was routinely used to avoid myocardial rewarming. With the introduction of warm myocardial preservation, the need for hypothermic perfusion was avoided and normothermic perfusion was popularized. ${ }^{2}$ There was concern, however, about the effects of normothermic perfusion on neurologic function and the possible deleterious effects of normothermic perfusion on the incidence of intraoperative stroke. ${ }^{3}$ This research was designed to study this relationship in a controlled setting.

An ongoing prospective, randomized National Institutes of Health-funded clinical trial was begun 
in February 1994 to study the influence of CPB perfusate temperature during coronary revascularization on factors that affect recovery. Initial efforts were dedicated to the study of neurologic function on the basis of the Mathew scale, ${ }^{4}$ as modified by Gelmers and associates, ${ }^{5}$ done before operation, at postoperative day 3 or 4 (before discharge from the hospital), and at 1 month by a neurologist blinded to perfusion temperature. Additional parameters regarding rapidity of recovery, such as time to extubation, amount of postoperative blood loss, creatine kinase (CK) MB generation, perioperative myocardial infarction, and postoperative length of stay, were measured for each patient. Tests done only after initiation of the study, in recent months, have included measurements of fibrinolytic activity.

\section{Methods}

This study randomly selected one of three perfusion temperatures for $\mathrm{CPB}$, cold $\left(20^{\circ} \mathrm{C}\right)$, tepid $\left(32^{\circ} \mathrm{C}\right)$, or warm $\left(37^{\circ} \mathrm{C}\right)$. There were 130 patients randomized for this report who were divided into 37 patients in the cold group, 50 in the tepid, and 43 in the warm. The study was approved by the Baystate Medical Center Institutional Review Board and is scheduled for completion in 1997. Informed consent was obtained from every patient. Randomization was accomplished by a computer-generated random number assignment. All patients were randomized in the operating room before the start of CPB into one of the three temperature protocols.

Warm CPB was conducted with perfusate temperature strictly at $37^{\circ} \mathrm{C}$, tepid at $32^{\circ}$, and cold at $20^{\circ} \mathrm{C}$. Only during rewarming from cold perfusion was deliberate hyperthermia $\left(39^{\circ} \mathrm{C}\right)$ used for any part of the rewarming process, and this never, in any case, exceeded a $10^{\circ} \mathrm{C}$ temperature gradient. If the perfusate was cooled (to either $32^{\circ} \mathrm{C}$ or $20^{\circ} \mathrm{C}$ ), then rewarming was initiated with the last distal anastomosis and was continued during the placement of proximal aorta-saphenous vein anastomoses with use of a side-biting aortic clamp or single aortic crossclamp with continued arrest if aortic atheroma was noted. Rewarming was accomplished with a perfusion temperature up to $39^{\circ} \mathrm{C}$ in patients in the cold group and up to $37^{\circ} \mathrm{C}$ in patients in the tepid group. Patients were weaned from CPB after the rectal or nasopharyngeal core temperature reached $36^{\circ} \mathrm{C}$. During cardioplegic arrest, core temperature reached $25^{\circ}$ to $28^{\circ} \mathrm{C}, 32^{\circ}$ to $33^{\circ} \mathrm{C}$, and $35^{\circ}$ to $36^{\circ} \mathrm{C}$ in the cold, tepid, and warm groups, respectively. The surgeons participating in the study were the entire the Baystate Medical Center cardiac surgical staff (R. M. E.; J. A. R.; J. E. F.; D. W. D.).

All patients having elective and urgent coronary revascularization with three or more bypass grafts (to ensure a bypass time in excess of 60 minutes) and younger than 76 years old (to reduce the risk of aortic, carotid, or intracerebral atherosclerosis as a factor) were candidates for the study. Of the 130 patients, $40.8 \%$ required elective and $59.2 \%$ required urgent operation (defined as requir- ing operation as soon as feasible during the same hospitalization). Exclusion factors were any neurologic disease history, poor ventricular function (left ventricular ejection fraction $<30 \%$ ), ongoing congestive heart failure, renal failure with serum creatinine level greater than $2.0 \mathrm{U} / \mathrm{L}$, or recent postinfarction angina. Patients in whom the postoperative neurologic examination could not be done, for example, because of mortality, reoperation, poor ventricular function and low output syndrome, multisystem organ failure, or refusal to undergo a follow-up examination, were excluded from the current study and are included in a separate report. Data for patients in whom a temperature protocol was not adhered to for any reason were reported with the patient's intent-to-treat group, for example, patients who required a cold perfusion temperature for achieving cardioplegic arrest, and are described separately.

Anesthesia was consistent across the three groups: premedication with lorazepam ( 1 to $2 \mathrm{mg}$ orally); induction with sufentanil ( 1 to $2 \mu \mathrm{g} / \mathrm{kg}$ intravenously) or fentanyl (5 to $15 \mu \mathrm{g} / \mathrm{kg}$ intravenously), midazolam $(0.05$ $\mathrm{mg} / \mathrm{kg}$ intravenously), and pancuronium $(0.15 \mathrm{mg} / \mathrm{kg}$ intravenously); and maintenance with sufentanil $(<4 \mu \mathrm{g} / \mathrm{kg}$ intravenously) or fentanyl ( $<20 \mu \mathrm{g} / \mathrm{kg}$ intravenously) and isoflurane titrated as tolerated (usually $0.5 \%$ to $1.0 \%$ ). Extubation occurred in the intensive care unit as rapidly as feasible with use of standard respiratory parameters. All patients had a rectal temperature greater than $36^{\circ} \mathrm{C}$ before extubation. No attempt was made to deliberately maintain paralysis or analgesic medication to extend the duration of intubation, but if shivering occurred during recovery, medication was occasionally necessary until full rewarming. "Fast track" recovery was attempted in every patient to accomplish an expeditious discharge from the hospital. ${ }^{6}$ Our fast track protocol used methylprednisolone $1 \mathrm{gm}$ intravenously before bypass and dexamethasone $4 \mathrm{mg}$ intravenously every 6 hours for four doses ( 24 hours) beginning immediately after operation. Steroid dose was reduced by $50 \%$ in patients with diabetes.

The adequacy of myocardial preservation was gauged by (1) documentation of perioperative infarction with electrocardiographic or CK-MB criteria, or both; (2) recording of inotrope use, and (3) measurement of daily CK-MB levels. CPB used a Cobe roller pump (Cobe Cardiovascular, Inc., Arvada, Colo.) and the Affinity hollow fiber membrane (Avecor Cardiovascular, Inc., Plymouth, Minn.). An $8.0 \mathrm{~mm}$ Sarns aortic arch cannula (Sarns/3M Health Care, Ann Arbor, Mich.) or a 20F DLP arterial cannula (DLP, Inc., Grand Rapids, Mich.) was used for aortic cannulation, and a $36 \mathrm{~F}$ dual-drainage Research Medical single venous return catheter (Research Medical, Inc., Midvale, Utah) was used for venous access. A DLP Gundry RCSP 15 F coronary sinus catheter was used for retrograde delivery of cardioplegic solution.

CBP was done at flow rates of $2 \mathrm{~L} / \mathrm{min}$ per square meter or greater when the patients underwent hypothermia and up to $3 \mathrm{~L} / \mathrm{min}$ per square meter during normothermia. In-line venous saturation was maintained at greater than $70 \%$ and perfusion pressures deliberately maintained between 60 and $80 \mathrm{~mm} \mathrm{Hg}$ with the use of phenylephrine for vasoconstriction and isoflurane for vasodilation. The hematocrit value was maintained at $20 \%$ or greater during 
Table I. Description of the Mathew scale

\begin{tabular}{lr}
\hline \multicolumn{1}{c}{ Category } & Score \\
\hline Consciousness & 8 \\
Orientation & 6 \\
Language & 23 \\
Strength & 20 \\
Reflexes & 3 \\
Sensation & 3 \\
Visual fields & 3 \\
Gaze palsy & 3 \\
Facial weakness & 3 \\
Disability & 28 \\
Total & 100 \\
\hline
\end{tabular}

the operation. Heparin was administered initially at 500 $\mathrm{u} / \mathrm{kg}$ body weight intravenously and CPB initiated only if the activated clotting time was greater than 600 seconds and the heparin level greater than $3 \mathrm{mg} / \mathrm{kg}$ body weight. Measurements of activated clotting time and heparin levels were repeated every 30 minutes during $\mathrm{CPB}$, and heparin was administered as necessary.

Myocardial preservation was by antegrade-retrograde blood cardioplegia with the use of $4: 1$ blood/crystalloid solution at the temperature chosen for the study. Cold preservation used $8^{\circ}$ to $10^{\circ} \mathrm{C}$ blood cardioplegic solution, whereas tepid preservation used $32^{\circ} \mathrm{C}$ and warm $37^{\circ} \mathrm{C}$ solution. Cardioplegic solution was administered in a retrograde fashion and through each completed vein graft intermittently during the procedure in a similar manner regardless of temperature. Aortic venting was used in every patient. Whenever the retrograde flow of blood cardioplegic solution interfered with visualization of the anastomoses, infusion of the solution was interrupted. When we analyzed the percent of arrest time, when cardioplegic solution was actually administered in the majority of patients, the result was $70 \%$ of the total arrest time, and at no time was cardioplegia interrupted for more than 10 minutes at a time. ${ }^{7}$ Hyperkalemia was avoided by using low-potassium cardioplegic solution $\left(\mathrm{K}^{+}=8\right.$ to $\left.10 \mathrm{mEq} / \mathrm{L}\right)$ except for induction of arrest $\left(\mathrm{K}^{+}\right.$ $=24 \mathrm{mEq} / \mathrm{L}$ ). Intraoperative hyperglycemia was avoided by sharply reducing crystalloid cardioplegic solution glucose administration such that the blood cardioplegic solution glucose levels were normoglycemic, with the blood/ crystalloid ratio at 4:1.

The primary end point of the study was neurologic function. This was quantitatively assessed in every patient with use of the Mathew scale (Table I). This is an accepted measure that was scored before operation, 3 to 4 days after operation but before discharge from the hospital, and at 1 month when the patient returned for follow-up examination. When any abnormality was detected, a cerebral computed tomographic (CT) scan was done, and if a cerebrovascular accident was diagnosed, a magnetic resonance imaging angiogram was completed. At operation, to rule out aortic atherosclerosis, epiaortic echocardiographic imaging was done in any patient for whom there was a suspicion of atheroma. If atherosclerosis was noted, the patient was included in the trial but specifics were noted as to the presence of aortic disease.

Measurements of fibrinolytic potential were studied in collaboration with Colorado Coagulation Consultants, Inc. (Denver, Colo.). Six studies were done: tissue plasminogen activator antigen ( $\mathrm{t}-\mathrm{PA}$ antigen), plasminogen activator inhibitor (PAI-1), plasma antithrombin (PAT) (previously termed antithrombin III), plasminogen, prekallikrein, and $\alpha_{2}$-antiplasmin. Each was done immediately before operation, at the completion of operation (on admission to the intensive care unit), and at 2, 4, 12, and 24 hours after operation. The tests required blood to be collected in $3.8 \%$ buffered citrate solution. Plasma was immediately separated and frozen at $-70^{\circ} \mathrm{C}$ and shipped on dry ice to Colorado Coagulation Consultants for analysis.

The specific techniques used for the hematologic studies were (1) for t-PA antigen (measured in nanograms per milliliter), the BioPool TintElize test (BioPool/Meditec, Inc., Ventura, Calif.), which uses the double antibody principle and enzyme-linked immunosorbent assay methods; (2) for PAI-1 (measured in units per milliliter), the BioPool Spectrolyse test (BioPool/Meditec), which is a two-stage, enzymatic assay; (3) for PAT (measured in percent activity), the Dade antithrombin III chromogenic assay, which measures the functional levels of PAT by an amidolytic method with use of a synthetic chromogenic substrate (Baxter Diagnostics, Inc., Deerfield, Ill.); (4) for plasminogen (measured in percent activity), the chromogenic assay that measures the functional levels of plasminogen by an amidolytic method with use of a synthetic chromogenic substrate (Pharmacia/Kabi, Franklin, Ohio); (5) for prekallikrein (measured in percent activity), the generally accepted one-stage activated partial thromboplastin time measurement (Colorado Coagulation Consultants); and (6) for $\alpha_{2}$-antiplasmin (measured in percent activity), the chromogenic assay that measures the functional levels in plasma by an amidolytic method with use of a synthetic substrate (Pharmacia/Kabi). Analyses were corrected in each instance for hemodilution by the following calculation: corrected concentration or activity $=$ (sample concentration or activity)(initial hematocrit)/ (sample hematocrit).

The data were analyzed in two ways: first, as the absolute change in activity, $X_{c}-X_{p}$, where $X_{c}$ is the corrected postoperative value and $X_{p}$ is the preoperative or baseline value, and second, as the relative change in activity, or percent change from baseline, $100\left(X_{c}-\right.$ $\left.\mathrm{X}_{\mathrm{p}}\right) / \mathrm{X}_{\mathrm{p}}$. In the first calculation, the measurement expressed either a concentration or percent activity. In the second, the calculation expressed a percent change. If the level increased during $\mathrm{CPB}$, the value was positive and if it decreased, the value was negative.

Statistical analysis and data collection. All patient data relative to recovery were collected prospectively by research assistants blinded to temperature. The data include the Society of Thoracic Surgeons database information, all atrial and ventricular arrhythmias (whether treated or not), time until extubation, blood collected after operation (whether reinfused or not), blood and blood products transfused, and postoperative length of stay. Temperature groups were compared with the use of 
Table II. Preoperative and intraoperative data on 130 National Institutes of Health patients

\begin{tabular}{lccc}
\hline & Cold & Tepid & Warm \\
\hline Preoperative data & & & 43 \\
No. of patients & 37 & $62.9 \pm 1.1$ & $60.5 \pm 1.3$ \\
Mean age (yr) & $65.9 \pm 1.3$ & $41-75$ & $45-74$ \\
Range of age (yr) & $47-75$ & 84.0 & 81.4 \\
Men (\%) & 67.6 & 36.0 & 23.3 \\
Diabetes (\%) & 21.6 & 64.0 & 65.1 \\
Hypertension (\%) & 54.1 & 8.0 & 0.0 \\
COPD (\%) & 8.1 & 52.0 & 37.2 \\
History of myocardial infarction (\%) & 45.9 & 56.0 & 51.2 \\
History of unstable angina (\%) & 59.5 & 24.0 & 20.9 \\
Left main disease (\%) & 29.7 & $51.9 \pm 1.3$ & $52.1 \pm 1.5$ \\
Mean ejection fraction (\%) & $51.6 \pm 1.5$ & & \\
Operative data & & $4.0 \pm 0.1$ & $3.9 \pm 0.1$ \\
No. of grafts (mean) & $3.8 \pm 0.1$ & 100 & 95.3 \\
Internal thoracic artery used (\%) & 100 & $141.7 \pm 5.5$ & $137.5 \pm 5.7$ \\
Mean CPB time (min) & $140.4 \pm 5.7$ & $86.5 \pm 4.2$ \\
Mean crossclamp time (min) & $84.0 \pm 3.8$ & $1672 \pm 9.2 \pm 4.2$ & $1945 \pm 118.1$ \\
Crystalloid cardioplegic solution (ml)* & $1672 \pm 120.2 \dagger$ & \\
\hline
\end{tabular}

*Amount of crystalloid cardioplegic solution: total cardioplegic volume is divided by 5 because blood is 4:1 crystalloid. $\dagger p<0.01$ by $t$ test.

simple descriptive statistics such as the mean plus or minus standard error and selected percentiles for some variables. Significance, defined at the $\alpha=0.05$ level, was determined by $\chi^{2}$ or Fisher's exact tests for association, Mantel-Haenszel $\chi^{2}$ tests for ordered trend across temperature groups for categorical variables, and by least squares or Kruskal-Wallis analysis of variance for continuous scale data. Nonparametric methods were used when the distributional assumptions were in doubt, particularly with the presence of extreme values, which skew the distribution and can result in large differences in error variances across groups. Values reported in the tables are means plus or minus standard errors, even when a nonparametric test was used to assess significance. When differences among the three groups were noted, pairwise $t$ tests (or a $t$ test approximation to Wilcoxon rank-sum statistic), with use of a Bonferroni correction for multiple comparisons, were used to determine where the differences lay.

A multivariate analysis of variance was used to evaluate differences across the temperature groups in change from preoperative values (absolute and percent change) for the six hematologic factors. Significance tests for differences among the temperature groups, as well as ordered trend across temperature, were conducted for each factor and across all factors.

\section{Results}

Preoperative and operative variables (Table II) were comparable among groups and only volume of cardioplegic solution differed significantly across temperature groups. Mean age was 61 to 66 years and ejection fraction was $52 \%$. All patients, except two, received internal thoracic artery grafts with the mean number of grafts near 4.0. Mean crossclamp time was 84 to 88 minutes in all three groups, and mean CPB time was 138 to 142 minutes, a reflection of how uniform the operative approach was regardless of temperature. As expected, the smallest volume of cardioplegic solution was administered in the cold group ( $p<0.01$ cold versus tepid; not significant cold versus warm).

Postoperative data (Table III) document differences, which do not, however, always reach significance. The highest mean weight gain, longest mean duration of intubation, and longest mean postoperative length of stay all occurred in the cold group, which is perhaps a reflection of longer intensive care unit stays necessitated by hypothermia. The analysis of variance on ranked values (Kruskal-Wallis) showed length of stay approaching significance across temperature $(p=0.09)$, and by pairwise comparison (Wilcoxon statistic) length of stay in the cold group was significantly longer than that in the tepid group $(p=0.02)$. Mean blood loss was least in the cold group but the difference did not reach significance. No patient in the cold group required reoperation because of bleeding whereas one patient in the tepid group and two in the warm group had to have a reoperation. Blood or blood product administration at any time during postoperative hospitalization took place in only $26 \%$ to $32 \%$ of patients, with no distinction as to group. There was no perioperative or late mortality in any group. The prevalence of atrial fibrillation, including transient, 
Table III. Postoperative data on 130 National Institutes of Health patients

\begin{tabular}{lccc}
\hline & Cold & Tepid & Warm \\
\hline No. of patients & 37 & 50 & 43 \\
Perioperative mortality (\%) & 0 & 0 & 0 \\
Peak postoperative weight gain (kg) & $4.9 \pm 2.7$ & $1.2 \pm 1.0$ & $1.8 \pm 0.6$ \\
Mean intubation time (hr) & $11.6 \pm 1.7$ & $10.4 \pm 0.9$ & $10.1 \pm 0.9$ \\
Peak postoperative CK-MB level (u/L) & $43.4 \pm 4.2^{*}$ & $39.4 \pm 3.2 \dagger$ & $33.5 \pm 2.6^{*}$ \\
Inotrope use (\%) & 32.4 & 34.0 & 23.3 \\
Intraaortic balloon pump (\%) & 2.7 & 2.0 & 7.0 \\
Postoperative length of stay (days) & $7.0 \pm 0.8^{*}$ & $5.2 \pm 0.2^{*}$ & $5.6 \pm 0.4$ \\
Discharge within 3-5 days (\%) & 56.8 & 72.0 & 65.1 \\
Range of length of stay (days) & $3-21$ & $3-9$ & $3-16$ \\
New focal deficit on postoperative neurologic & $18.9^{*}$ & $2.0^{*}$ & 9.3 \\
$\quad$ examination (\%) & & 8.1 & 0.0 \\
CT scan-confirmed stroke (\%) & $831.2 \pm 56.8$ & $963.8 \pm 82.9$ & $912.5 \pm 98.9$ \\
Blood loss (ml) & 67.6 & 74.0 & 67.4
\end{tabular}

* $p<0.05$ by Fisher's exact test or $t$ test.

$\dagger$ The only patient with a perioperative infaretion is omitted from this table but not from the statistical analysis of the data.

was $20 \%$ to $28 \%$ in the three groups, and atrial fibrillation that necessitated any specific therapy other than digoxin, which was used routinely, was $18.9 \%, 14.0 \%$, and $9.3 \%$ in the cold, tepid, and warm groups, respectively (not significant). Ventricular tachycardia (defined as 3 or more ventricular premature complexes together) occurred in $16 \%$ to $28 \%$ of patients with no significant difference among groups.

Myocardial injury as reflected by perioperative infarction occurred in only one patient in the tepid group. Mean CK-MB generation was least in the warm group $(33.5 \mathrm{u} / \mathrm{L}$ warm versus $43.4 \mathrm{u} / \mathrm{L}$ cold, $p<0.05)$. Inotrope use, a reflection of myocardial function, was also least in the warm group (23\% warm, 34\% tepid, $32 \%$ cold), but this difference was not significant. Remarkably, the use of an intraaortic balloon at any time was highest in the warm group (three patients), with one each in the cold and tepid groups. Any profound influence of temperature on myocardial preservation was not clearly apparent in this study.

Neurologic function. One hundred sixteen of the 130 patients completed all three neurologic examinations (Table IV). Fourteen patients did not complete one of the postoperative neurologic examinations. Two underwent reoperation because of bleeding, two had low-output syndrome, and two had prolonged intubations that prevented full neurologic examinations as scheduled. Eight additional patients missed a follow-up examination. None of these 14 patients had an adverse neurologic outcome, but they were excluded from the Mathew scale analysis. The distribution of these patients across groups was three in the cold, five in the tepid, and six in the warm group. One patient was randomized to the cold group but received the warm method because of physician preference. There was no Mathew scale deterioration in this patient. No other patient changed temperature groups.

There was a uniform decrease in Mathew scale findings from preoperative to postoperative scores across all groups $(p=0.0001)$ and from preoperative to 1-month follow-up ( $p=0.0001$ ) (Table V). The distinction among groups was not significant $(p>0.5)$. There was significant improvement in scores between early postoperative and 1-month follow-up examinations $(p=0.0001)$, which again was not different among groups. The percent of patients with no deterioration in Mathew scale scores at any time after operation was $31.4 \%$, $28.9 \%$, and $30.6 \%$ in the cold, tepid, and warm groups, respectively. This difference was not significant.

The Mathew scale (Table I) provides a means suggesting the diagnosis of stroke associated with coronary revascularization by grading the severity of neurologic dysfunction and quantitating lateralizing signs of motor, visual, and reflex function. When there was any evidence of localizing neurologic dysfunction consistent with cerebrovascular accident, no matter how mild, a CT scan was obtained. Twelve patients, seven in the cold group $(18.9 \%)$, one in the tepid $(2.0 \%)$, and four in the warm $(9.3 \%)$, were referred for diagnostic CT scanning (Table III). The association of bypass temperature with abnormal neurologic examina- 
Table IV. Mathew scale summary

\begin{tabular}{lrr}
\hline & $N$ & Mean $\pm S E M$ \\
\hline Preop-test & & \\
Cold & 35 & $99.0 \pm 0.23$ \\
Tepid & 45 & $99.4 \pm 0.17$ \\
Warm & 36 & $99.0 \pm 0.52$ \\
All & 116 & $99.1 \pm 0.19$ \\
Postop. test & & \\
Cold & 35 & $94.6 \pm 0.93$ \\
Tepid & 45 & $96.2 \pm 0.67$ \\
Warm & 36 & $95.1 \pm 1.12$ \\
All & 116 & $95.4 \pm 0.52$ \\
Follow-up test & & \\
Cold & 35 & $97.7 \pm 0.61$ \\
Tepid & 45 & $97.6 \pm 0.54$ \\
Warm & 36 & $98.1 \pm 0.66$ \\
All & 116 & $97.7 \pm 0.34$
\end{tabular}

At no time was there a significant difference between temperature groups. $S E M$, standard error of the mean.

tion results prompting CT scanning was significant across the different temperatures ( $p=0.02$ by Fisher's exact test). CT scanning confirmed a cerebrovascular accident in only three patients in the cold group $(8.1 \%)$, none in the tepid group $(0 \%)$, and two in the warm group $(4.7 \%)$. This difference was not significant ( $p=0.10$ by Fisher's exact test). Magnetic resonance angiography was done in all five patients with positive findings on CT scan and documented no intracerebral or extracerebral atherosclerosis as a cause for cerebrovascular accident. This supports embolization as the most likely cause. For purposes of comparing this study with less critical reports of postoperative neurologic dysfunction, of this fivepatient cerebrovascular accident group, only two had a grossly apparent stroke that would have been noted by routine examination (as distinguished from a sophisticated preoperative and postoperative neurologic examination) and all five had complete recovery on long-term (3 month) follow-up. In three patients with cerebrovascular accident, neither the patients nor their families were aware of any neurologic dysfunction.

An atherosclerotic ascending aorta was noted before bypass by epiaortic imaging in two patients, both in the warm group. Measures were taken, such as femoral arterial cannulation and single aortic clamping, to prevent embolization, but despite this, one of the two patients had a cerebrovascular accident, which was small and from which recovery was complete. This same patient is included as one of the five in the overall series.
Table V. Mathew scale difference

\begin{tabular}{lrc}
\hline & $N$ & Mean $\pm S E M$ \\
\hline Postop.-preop. difference & & \\
Cold & 35 & $-4.3 \pm 0.98^{*}$ \\
Tepid & 45 & $-3.3 \pm 0.67^{*}$ \\
Warm & 36 & $-3.9 \pm 1.08^{*}$ \\
All & 116 & $-3.7 \pm 0.51^{*}$ \\
Follow-up-preop. difference & & \\
Cold & 35 & $-1.3 \pm 0.64^{*}$ \\
Tepid & 45 & $-1.8 \pm 0.51^{*}$ \\
Warm & 36 & $-0.9 \pm 0.33^{*}$ \\
All & 116 & $-1.4 \pm 0.29^{*}$ \\
Follow-up-postop. difference & & \\
Cold & 35 & $+3.0 \pm 0.98^{*}$ \\
Tepid & 45 & $+1.4 \pm 0.70^{*}$ \\
$\quad$ Warm & 36 & $+2.9 \pm 1.17^{*}$ \\
All & 116 & $+2.4 \pm 0.54^{*}$ \\
\hline Minus sign indicates a decrease and plus sign an increase in Mathew scale \\
score. SEM, standard error of the mean. \\
*A significant difference with $p=0.0001$ for postoperative to preoperative \\
comparison, follow-up to preoperative comparison, and follow-up to \\
postoperative comparison, with no difference between groups.
\end{tabular}

Fibrinolytic potential. The volume of postoperative blood loss was not significantly different across the three temperature groups (Table III) (mean = $831 \mathrm{ml}$ cold, $964 \mathrm{ml}$ tepid, $913 \mathrm{ml}$ warm) although it was clearly least in the cold group. Additionally, no patient in the cold group required reoperation because of bleeding. If the three patients (one in the tepid and two in the warm group) with reoperations are deleted from the blood loss analysis, the volume of blood collected after operation was similar among groups ( $831 \pm 57 \mathrm{ml}$ cold, $906 \pm 61 \mathrm{ml}$ tepid, $796 \pm$ $58 \mathrm{ml}$ warm). No antifibrinolytics were used in the study (for example, $\epsilon$-aminocaproic acid, tranexamic acid, or aprotinin). Although hematologic data were collected before CPB and after CPB at $0,2,4,12$, and 24 hours after operation, the only data to be discussed in depth will be measurements taken immediately after CPB. In this analysis of CPB temperature, fibrinolytic assay differences were most evident at completion of CPB.

Six different hematologic features were measured, each having a role in CPB-induced fibrinolysis or fibrinolytic potential. A comparison was done for each measurement in the preoperative determination for differences across temperature groups and, as expected, there was no significant difference found. Each postoperative value was corrected for hemodilution and the actual differences between the postoperative and preoperative levels are presented in Table VI. The determination of a percent change from preoperative values is described in Table VII. 
Table VI. Hematologic summary data for difference between postoperative and preoperative values

\begin{tabular}{|c|c|c|c|c|c|}
\hline Test & Temperature & $N$ & $M e a n \pm S E M$ & $A N O V A$ & Trend \\
\hline \multirow[t]{4}{*}{ t-PA antigen $(\mathrm{ng} / \mathrm{ml})$} & Cold & 9 & $+18.56 \pm 3.29^{*}$ & & \\
\hline & Tepid & 6 & $+11.32 \pm 3.12^{*}$ & & \\
\hline & Warm & 10 & $+15.96 \pm 3.22^{*}$ & 0.372 & 0.560 \\
\hline & All & 25 & $+15.78 \pm 1.91^{*}$ & & \\
\hline \multirow[t]{4}{*}{ PAI-1 (u/ml) } & Cold & 9 & $+24.09 \pm 5.75^{*}$ & & \\
\hline & Tepid & 6 & $+16.07 \pm 7.21$ & & \\
\hline & Warm & 10 & $+27.78 \pm 5.71^{*}$ & 0.451 & 0.654 \\
\hline & All & 25 & $+23.64 \pm 3.51^{*}$ & & \\
\hline \multirow[t]{4}{*}{ PAT (\% activity) } & Cold & 9 & $-1.93 \pm 3.40$ & & \\
\hline & Tepid & 6 & $+6.38 \pm 8.90$ & & \\
\hline & Warm & 10 & $-11.55 \pm 4.85^{*}$ & 0.098 & 0.192 \\
\hline & All & 25 & $-3.78 \pm 3.31$ & & \\
\hline \multirow[t]{4}{*}{ Plasminogen (\% activity) } & Cold & 9 & $+3.04 \pm 5.42$ & & \\
\hline & Tepid & 6 & $-6.71 \pm 5.52$ & & \\
\hline & Warm & 10 & $-19.61 \pm 5.33^{*}$ & 0.018 & 0.0053 \\
\hline & All & 25 & $-8.36 \pm 3.66^{*}$ & & \\
\hline \multirow[t]{4}{*}{ Prekallikrein (\% activity) } & Cold & 9 & $-2.47 \pm 6.23$ & & \\
\hline & Tepid & 6 & $-8.36 \pm 7.29$ & & \\
\hline & Warm & 10 & $-27.56 \pm 6.39^{*}$ & 0.025 & 0.0092 \\
\hline & All & 25 & $-13.92 \pm 4.34^{*}$ & & \\
\hline \multirow[t]{4}{*}{$\alpha_{2}$-Antiplasmin ( $\%$ activity) } & Cold & 9 & $+5.11 \pm 3.80$ & & \\
\hline & Tepid & 6 & $-1.14 \pm 7.03$ & & \\
\hline & Warm & 10 & $-12.75 \pm 8.31$ & 0.165 & 0.064 \\
\hline & All & 25 & $-3.54 \pm 4.14$ & & \\
\hline
\end{tabular}

$S E M$, Standard error of the mean; $A N O V A$, analysis of variance.

*Significant change from preoperative value, $p<0.05$.

The differences across temperature groups were tested for significance by analysis of variance. In addition, a test for linear trend across the three temperature groups ordered as cold-tepid-warm was evaluated. These results are reported in Tables VI and VII.

Among the studies done, a significant temperature-dependent difference was noted only in the decrease in plasminogen and prekallikrein levels with the greatest decrease occurring in patients in the warm group and the least in patients in the cold group. The analysis of variance $p$ value was 0.018 for plasminogen and 0.025 for prekallikrein with a trend analysis $p$ value of 0.0053 for plasminogen and 0.0092 for prekallikrein. The percent change from the preoperative value between groups was also only significantly different across the three groups in plasminogen and prekallikrein levels with similar $p$ values for both analysis of variance and the linear trend. The fact that both analyses were statistically consistent underscores the validity of the data.

A multivariate analysis across the six factors measured for an overall temperature effect was not significant ( $\mathrm{F}$ approximations to Hotelling-Lawley trace, Roy's largest root). Although the warm group in general had the greatest negative score, this was not consistent across all factors and the ordering of cold and tepid was also not consistent ( $p=0.258$ for linear trend effect).

As described in Tables VI and VII, neither all groups nor all studies had postoperative findings that were significantly different from preoperative control findings. The endothelial-generated fibrinolytic t-PA antigen and antifibrinolytic PAI-1 showed uniform changes across all temperature groups with a significant increase in postoperative t-PA antigen and PAI-1 levels. With regard to PAT, plasminogen, and prekallikrein only the warm group had a significant decrease from preoperative values, whereas the other two groups either showed a small insignificant increase or values that remained near the preoperative level.

\section{Discussion}

The impetus of this study was to determine whether perfusion temperature could be a factor in recovery from CPB. Clearly, in only a minority of operations is there an overriding reason for choosing one temperature over any other, as for example in hypothermic circulatory arrest. When myocardial hypothermia was routinely favored for preservation, systemic hypothermia seemed appropriate. Now 
Table VII. Hematologic summary data for percent difference from preoperative values

\begin{tabular}{|c|c|c|c|c|c|}
\hline Test & Temperature & $N$ & Mean $\pm S E M$ & $A N O V A$ & Trend \\
\hline \multirow[t]{4}{*}{ t-PA antigen (ng/ml) } & Cold & 9 & $+146.9 \pm 25.7^{*}$ & \multirow{4}{*}{0.633} & \multirow{4}{*}{0.566} \\
\hline & Tepid & 6 & $+119.7 \pm 41.3^{*}$ & & \\
\hline & Warm & 10 & $+180.0 \pm 51.4^{*}$ & & \\
\hline & All & 25 & $+153.6 \pm 24.2^{*}$ & & \\
\hline \multirow[t]{4}{*}{ PAI-1 (u/ml) } & Cold & 9 & \multirow{4}{*}{\multicolumn{3}{|c|}{$\begin{array}{l}\text { Normal PAI }-1 \text { is } 0-15 \mathrm{u} / \mathrm{ml} \text {. To avoid an unrealistic percent calculation } \\
\text { based on an increase from } 0 \text {, these data are reported only as the actual } \\
\text { difference from preoperative values in Table VI. }\end{array}$}} \\
\hline & Tepid & 6 & & & \\
\hline & Warm & 10 & & & \\
\hline & All & 25 & & & \\
\hline \multirow[t]{4}{*}{ PAT (\% activity) } & Cold & 9 & $-1.7 \pm 3.8$ & \multirow{4}{*}{0.120} & \multirow{4}{*}{0.199} \\
\hline & Tepid & 6 & $+6.5 \pm 9.6$ & & \\
\hline & Warm & 10 & $-12.2 \pm 5.4$ & & \\
\hline & All & 25 & $-3.9 \pm 3.6$ & & \\
\hline \multirow[t]{4}{*}{ Plasminogen ( $\%$ activity) } & Cold & 9 & $+6.1 \pm 6.2$ & \multirow{4}{*}{0.016} & \multirow{4}{*}{0.0046} \\
\hline & Tepid & 6 & $-5.0 \pm 6.2$ & & \\
\hline & Warm & 10 & $-18.1 \pm 5.0^{*}$ & & \\
\hline & All & 25 & $-6.2 \pm 3.9$ & & \\
\hline \multirow[t]{4}{*}{ Prekallikrein (\% activity) } & Cold & 9 & $-1.4 \pm 5.7$ & \multirow{4}{*}{0.023} & \multirow{4}{*}{0.0085} \\
\hline & Tepid & 6 & $-6.6 \pm 7.0$ & & \\
\hline & Warm & 10 & $-24.1 \pm 5.4^{*}$ & & \\
\hline & All & 25 & $-11.7 \pm 3.9^{*}$ & & \\
\hline \multirow[t]{4}{*}{$\alpha_{2}$-Antiplasmin ( $\%$ activity) } & Cold & 9 & $+5.0 \pm 3.3$ & \multirow{4}{*}{0.180} & \multirow{4}{*}{0.070} \\
\hline & Tepid & 6 & $-0.9 \pm 7.1$ & & \\
\hline & Warm & 10 & $-11.0 \pm 7.4$ & & \\
\hline & All & 25 & $-2.8 \pm 3.8$ & & \\
\hline
\end{tabular}

SEM, Standard error of the mean; $A N O V A$, analysis of variance.

*Significant change from preoperative value, $p<0.05$.

that myocardial hypothermia is no longer an issue, systemic perfusion temperature becomes a point of obvious discussion. As evident in this study, myocardial and cardioplegic solution temperatures had no dramatic effect on either myocardial preservation or the prevalence of arrhythmias (atrial or ventricular) and do not deserve to dictate a specific perfusion temperature. The CK-MB levels, although higher in cold-group than warm-group patients, were low and not consistent with myocardial infarction. CK-MB levels in the tepid group were between those in the cold and warm groups.

In this era of shrinking health care funding, the rapidity of postoperative recovery from anesthesia and extubation and appropriate shortening of intensive care unit stays have become subjects of more than passing interest. As shown by our own group ${ }^{6}$ and by Westaby and associates, ${ }^{8}$ early extubation is cost-effective and expedites recovery. Maintaining intensive care only for a short interval is clearly important for a host of reasons and can be more easily accomplished if significant hypothermia is avoided. As shown in this study, although the difference was not statistically significant, reduced intubation time was temperature dependent and a sig- nificantly longer postoperative length of stay was associated with hypothermic perfusion.

Neurologic function. Patients in all three temperature groups had lower Mathew scale scores at the immediate postoperative evaluation. There was no difference among groups and there was significant improvement at 1-month follow-up, again with no apparent difference among groups (Table V). The percentage of patients with any decrease in score at any time after operation was $68.6 \%, 71.1 \%$, and $69.4 \%$ in the cold, tepid, and warm groups, respectively, which was not significantly different among groups. This is consistent with the findings of other investigators who have reported postoperative neuropsychologic impairment in as many as $79 \%$ of patients, ${ }^{9}$ the cause of which is clearly multifactorial. Insofar as microemboli during $\mathrm{CPB}$ have been implicated, there would be, on the surface at least, reason to suspect hypothermic $\mathrm{CPB}$ to be neuroprotective. ${ }^{3,10,11}$ This was not the case, however, in other studies ${ }^{10,12}$ or in the present one, which have clearly shown no improved neurologic outcome with hypothermic perfusion.

The consequence of cold perfusion, in fact, is to lead to a significantly higher prevalence of abnormal 
neurologic examinations prompting $\mathrm{CT}$ scanning (18.9\% cold group versus $2.0 \%$ tepid and $9.3 \%$ warm, $p=0.02$ ). Although not associated with CT scan abnormalities in most patients, there remained a higher cerebrovascular accident prevalence in the cold group than in the tepid or warm groups $(8.7 \%$ cold, $0 \%$ tepid, $4.7 \%$ warm: difference not significant, $p=0.10$ ). The explanation for this seeming contradiction with the cold group having worse central nervous system function is speculative at best. A randomized, prospective trial was conducted through the University of Toronto and reported by Wong and associates ${ }^{10}$ in 1992. The investigators with a total of 34 patients found no new focal deficits in any of the groups but neuropsychologic test results were better in the warm group with one of seven tests reaching significance for warm versus cold (trail-making test A, $p=0.023$ ). There was no explanation offered for why function should have been better in the warm group. Singh, Bert, and Feng ${ }^{12}$ performed a retrospective analysis in which findings in a large number of patients in a warm group (2383) were compared with findings in a preceding cold group (1605), and they found a lower rate (difference not significant) of neurologic complications in the warm group. Again, no explanation was given for this finding. Finally, Martin and associates $^{3}$ reported on a randomized, prospective trial done at Emory University that documented a significantly higher prevalence of neurologic injury in the warm group. This was attributed to transient cerebral hypoperfusion, hyperglycemia, and microembolization during $\mathrm{CPB}$, which is more damaging in the normothermic setting.

It is the latter study that perhaps provides the most insight into this problem. The Emory group presented this paper at the Society of Thoracic Surgeons' annual meeting in $1993 .^{3}$ The oral presentation described in detail the actual mechanics of $\mathrm{CPB}$, specifically the perfusate temperature, which was not elaborated on in the published paper. The patients treated with a warm method were maintained deliberately at a core temperature near $37^{\circ} \mathrm{C}$ and perfusion was therefore hyperthermic (usually $39^{\circ} \mathrm{C}$ and never higher than $40^{\circ} \mathrm{C}$ ). ${ }^{13}$ It is the contention of our investigative group that hyperthermia is deleterious and contributes to central nervous system dysfunction. Prolonged hyperthermia, as during an entire CPB period, is simply prolonging the risk of central nervous system injury. Cold perfusion similarly is usually associated with rewarming hyperthermia and may thereby also lead to central nervous system dysfunction. ${ }^{14,15}$ This proposition is difficult, if not impossible, to prove but is supported by others, ${ }^{15}$ with a finding by the Mayo Clinic group of cerebral venous desaturation in $50 \%$ of patients during hyperthermic rewarming after standard hypothermic CPB. ${ }^{14}$

The results of neuropsychologic tests, such as those from the trail-making test, which Wong and associates ${ }^{10}$ found more depressed in cold-group patients, may indicate slower recovery from hypothermic rather than normothermic CPB without apparent central nervous system pathologic occurrences. Indeed, Townes and associates ${ }^{16}$ found neurobehavioral outcomes depressed in patients treated with hypothermia with resolution over time in the majority. The rapidity and normality of mentation can clearly be depressed by CPB, particularly when hypothermia is used, without apparent anatomic changes and ultimately complete recovery.

Fibrinolytic potential. The schematic for CPBinduced fibrinolysis is depicted in Fig. 1. Blood during $\mathrm{CPB}$ is in contact with foreign surfaces, which leads to activation of factor XII. ${ }^{17}$ Activated factor XII converts plasminogen to plasmin. This is accelerated by the contact conversion of prekallikrein to kallikrein, a consequence of heparin and $\mathrm{CPB} .{ }^{17-19}$ Endothelial cell perturbations also occur during $\mathrm{CPB}$, ischemic stress, and cardioplegia and contribute to generation of both a fibrinolytic or antithrombotic response, t-PA, and an antifibrinolytic or prothrombic response, PAI-1. ${ }^{20-22}$ These function in a fibrinolytic pathway to both stimulate and inhibit fibrinolysis. When both t-PA and PAI-1 are generated, the former is more physiologically dominant. ${ }^{23}$ Valen and associates ${ }^{22}$ measured t-PA and PAI-1 levels both during and after CPB. Although they found t-PA antigen levels to increase both during and after CPB, PAI-1 activity only increased after CPB. In the present study, measurements were made only after $\mathrm{CPB}$ and the results were similar to those reported by Valen and associates. ${ }^{22}$ There is a clear increase in fibrinolytic potential as seen with $\mathrm{t}$-PA generation during $\mathrm{CPB}$, which is the more dominant enzyme and is offset in part by PAI-1 release after operation. ${ }^{22,23}$ The changes in these agonist/antagonists were not temperature dependent but occurred uniformly across all temperatures. This implies comparable endothelial stimulation at all three temperatures.

Plasminogen is degraded to plasmin in the process of promoting fibrinolysis. In this study, plasminogen levels decreased significantly as a percent of 


\section{FIBRINOLYTIC POTENTIAL}

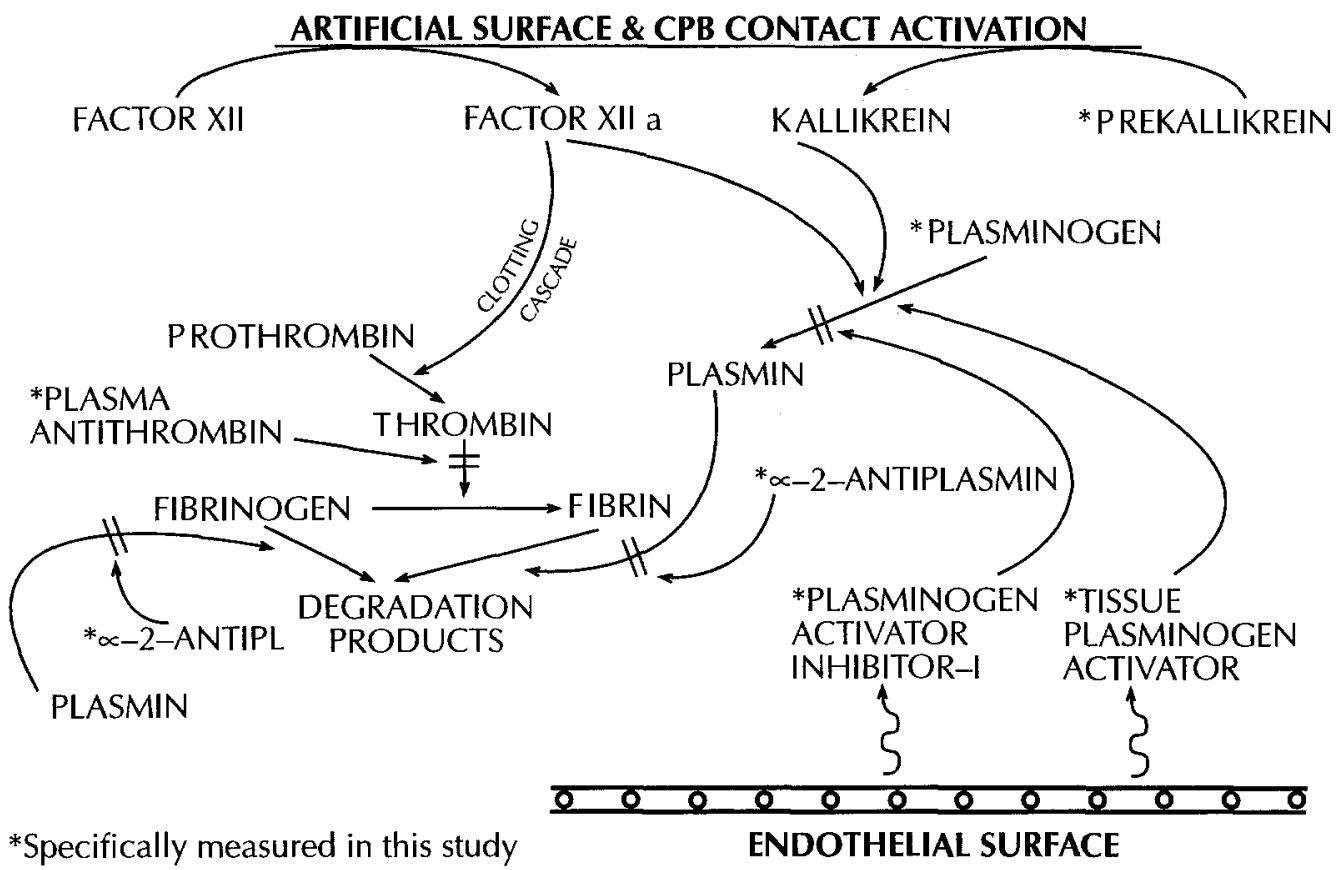

Fig. 1. Schematic for fibrinolytic potential occurring during CPB is illustrated. Blood and artificial surface contact lead to activation of Hageman factor (factor XII) and conversion of prekallikrein to kallikrein. Both serve to degrade plasminogen into plasmin. Activated factor XII, through its initiation of clotting cascade promotes formation of thrombin from prothrombin. Disturbances affecting endothelial cell, such as myocardial ischemia and cardioplegia, promote formation of t-PA, which functions to support degradation of plasminogen to plasmin. An antifibrinolytic, PAI-1, is also formed from endothelium, and functions to interrupt degradation of plasminogen. Plasmin, in turn, acts on fibrinogen and fibrin to form degradation products, which are end stage of fibrinolysis. Interfering with activity of plasmin is $\alpha_{2}$-antiplasmin $(\alpha-2-A N T I P L)$. PAT interrupts activity of thrombin in forming fibrin from fibrinogen. Specifically measured in this analysis were studies marked with asterisk: t-PA antigen, PAI-1, PAT, plasminogen, prekallikrein and $\alpha_{2}$-antiplasmin.

preoperative control values only in the warm group $(-18 \% \pm 5 \% ; p=0.005)$. The changes in the tepid $(-5 \% \pm 6 \%)$ and cold $(+6 \% \pm 6 \%)$ groups were not significant. This finding documents a significant difference in fibrinolysis with only warm perfusion promoting a significant degradation of plasminogen. The results with prekallikrein are similar with no significant difference noted in the cold or tepid groups but a significant decrease $(-24 \% \pm 5 \%)$ in the warm group $(p=0.002)$. This finding, together with a significant ordering across temperature groups for both quantitative measures of fibrinolysis (plasminogen and prekallikrein), supports the conclusion that artificial surface-induced fibrinolysis is temperature dependent. This study has shown normothermic perfusion to lead to increased fibrinolytic potential. The tests that documented this were increased t-PA antigen, decreased PAT, lowest plasminogen, lowest prekallikrein, and lowest $\alpha_{2}$-antiplasmin in normothermic patients. Neither tepid nor cold treatment had the same results. Whether this is only a result of increased surface activation of fibrinolysis or of a combination of factors in the normothermic group including increased endothelial stimulation cannot be definitively stated. However, this study does not have any data to support an increased endothelial component to normothermic bypass. Of interest in this regard is the finding of reoperation because of bleeding being necessary in two warm-group, one tepid-group, and no coldgroup patients.

Conclusion. There is a detriment to both cold and warm perfusion that is manifested by an increase in neurologic dysfunction in patients receiv- 
ing cold perfusion and an increase in fibrinolytic potential in patients receiving warm perfusion. Myocardial preservation temperature should not dictate perfusion temperature. The common practice of allowing the perfusate temperature to drift toward room temperature is most consistent with tepid perfusion. Rewarming during the latter phase of perfusion should not be hyperthermic or at least should be limited to a temperature close to $37^{\circ} \mathrm{C}$. If hyperthermia is necessary for rewarming, it should be for as short an interval as possible, with recognition of the inherent dangers.

\section{REFERENCES}

1. Lichtenstein SV, Ashe KA, El-Dalati H, Cusimano RJ, Panos A, Slutsky AS. Warm heart surgery. J Thorac Cardiovasc Surg 1991;101:269-74.

2. Christakis GT. Normothermic cardiopulmonary bypass. Ann Thorac Surg 1993;56:202-3.

3. Martin TD, Craver JM, Gott JP, et al. Prospective, randomized trial of retrograde warm blood cardioplegia: myocardial benefit and neurologic threat. Ann Thorac Surg 1994;57:298304.

4. Mathew NT, Rivera VM, Meyer JS, Charney JZ, Hartmann A. Double-blind evaluation of glycerol treatment in acute cerebral infarction. Lancet 1972;2:1327-9.

5. Gelmers HJ, Gorter $\mathrm{K}$, de Weerdt CJ, Wiezer HJ. A controlled trial of nimodipine in acute ischemic stroke. N Engl J Med 1988;318:203-7.

6. Engelman RM, Rousou JA, Flack JE III, et al. Fast-track recovery of the coronary bypass patient. Ann Thorac Surg 1994:58:1742-6.

7. Rousou JA, Engelman RM, Flack JE III, Deaton DW, Rifkin R, Elmansoury A. Does interruption of normothermic cardioplegia have adverse effects on myocardium: a retrospective and prospective clinical evaluation. Cardiovasc Surg 1995;3:587-93.

8. Westaby S, Pillai R, Parry A, et al. Does modern cardiac surgery require conventional intensive care? Eur $\mathbf{J}$ Cardiothorac Surg 1993;7:313-8.

9. Shaw PJ, Bates D, Cartlidge NEF, et al. Neurologic and neuropsychological morbidity following major surgery: comparison of coronary artery bypass and peripheral vascular surgery. Stroke 1987;18:700-7.

10. Wong BI, McLean RF, Naylor CD, et al. Central-nervoussystem dysfunction after warm or hypothermic cardiopulmonary bypass. Lancet 1992;339:1383-4.

11. Pugsley W, Klinger L, Paschalis C, Treasure T, Harrison M, Newman S. The impact of microemboli during cardiopulmonary bypass on neuropsychological functioning. Stroke 1994; 25:1393-9.

12. Singh AK, Bert AA, Feng WC. Neurological complications during myocardial revascularization using warm-body, coldheart surgery. Eur J Cardiothorac Surg 1994;8:259-64.

13. Mora CT, Henson MB, Weintraub WS, et al. The effect of temperature management during cardiopulmonary bypass on neurologic and neuropsychologic outcomes in patients undergoing coronary revascularization. J Thorac Cardiovasc Surg 1996;112:514-22.
14. Cook DJ, Orszulak TA, Daley RC, Buda DA. Cerebral hyperthermia during cardiopulmonary bypass in adults. $\mathbf{J}$ Thorac Cardiovasc Surg 1996;111:268-9.

15. Murkin JM. The role of CPB management in neurobehavioral outcomes after cardiac surgery. Ann Thorac Surg 1995;59:1308-11.

16. Townes BD, Bashein G, Hornbein TF, et al. Neurobehavioral outcomes in cardiac operations. J Thorac Cardiovasc Surg 1989;98:774-82.

17. Holloway DS, Summaria L, Sandesara J, Vagher JP, Alexander JC, Caprini JA. Decreased platelet number and function and increased fibrinolysis contribute to postoperative bleeding in cardiopulmonary bypass patients. Thromb Haemost 1988;59:62-7.

18. Kongsgaard UE, Smith-Erichsen N, Geiran O, Amundsen E, Mollnes TE, Garred P. Different activation patterns in the plasma kallikrein-kinin and complement systems during coronary bypass surgery. Acta Anaesthesiol Scand 1989;33: 343-7.

19. Burman JF, Chung HI, Lane DA, et al. Role of factor XII in thrombin generation and fibrinolysis during cardiopulmonary bypass. Lancet 1994;344:1192-3.

20. Tanaka K, Takao M, Yada I, Yuasa H, Kusagawa M, Deguchi K. Alterations in coagulation and fibrinolysis associated with cardiopulmonary bypass during open heart surgery. J Cardiothorac Anesth 1989;3:181-8.

21. Gram J, Janetzko T, Jespersen J, Bruhn HD. Enhanced effective fibrinolysis following the neutralization of heparin in open heart surgery increases the risk of post-surgical bleeding. Thromb Haemost 1990;63:241-5.

22. Valen G, Eriksson E, Risberg B, Vaage J. Fibrinolysis during cardiac surgery. Eur J Cardiothorac Surg 1994;8:324-30.

23. Zilla P, von Oppell U, Deutsch $\mathbf{M}$. The endothelium: a key to the future. J Card Surg 1993;8:32-60.

\section{Discussion}

Dr. Robert A. Guyton (Atlanta, Ga.). We performed a prospective, randomized trial of warm heart operation versus moderate hypothermia, which was published in 1994. Our warm group had a target bladder temperature of $37^{\circ} \mathrm{C}$, and hyperthermic arterial perfusion pressures with a maximum temperature of $38.5^{\circ} \mathrm{C}$ were necessarily used. Our conclusion was similar to the current conclusion. Hyperthermic arterial perfusion and consequent possible cerebral hyperthermia may be an important operative factor in the cause of neurologic injury. There are now nice studies in the neuroscience literature that examine the release of excitatory neurotransmitters and neurologic injury in response to temporary ischemia. Considerable neuroprotection is conferred with only $2^{\circ}$ or $3^{\circ}$ of hypothermia; that is, $33^{\circ} \mathrm{C}$ is better than $37^{\circ} \mathrm{C}$. These same studies have shown a dramatic negative effect of hyperthermia. On the basis of our studies, the Toronto work, the current study, and the neuroscience studies, we believe that tepid temperatures are neuroprotective, normothermia is not neuroprotective, and hyperthermic perfusate temperatures increase neurologic damage.

I have two questions for the authors regarding this superb study. First, I understand the difference that is suggested between the tepid and warm groups with regard to clinical neurologic deficits. I am uncomfortable, however, with the implication that hyperthermic perfusion 
fully explains the deficit rate in the cold group. The brain is hyperthermic only for a short time. In our own study we used hyperthermic warming perfusate in the cold group without observing a high neurologic deficit rate. Is there another reason for the deficits? For example, in the cold group, the average age was 66 years and in the warm group the average age was only 61 , which is not a significant difference, but perhaps was a contributing difference.

Finally, our study was terminated by our safety committee when we demonstrated a neurologic deficit rate with a $p$ value of 0.02 . Because the authors have demonstrated an increased neurologic deficit rate or neurologic abnormality rate in the cold group with a $p$ value of 0.01 , do they plan to continue the study with inclusion of the cold group or do they plan to eliminate the cold group as they continue the study?

Dr. Engelman. With regard to the question raised by concerns about cold perfusion, we now are aware of the risks of hyperthermic perfusion and are clearly limiting our hyperthermic perfusion to a much shorter interval during the bypass run with instructions given to our perfusionists as to what is necessary.

With regard to the concerns raised about neurodysfunction, we raised this issue with our institutional review board, and the decision made was based on the absolute finding of cerebrovascular accident, not just subtle neurologic dysfunction, which clearly is transient and may be more a subjective finding on the part of the neurologist rather than the objective finding that we have when we find a cerebrovascular accident. With the CT scan as the gold standard for determination of neurologic abnormality, we believe that there was no significant difference between the various groups, and therefore the institutional review board has thought that it would not be necessary at this time to terminate the study.

Dr. Thomas A. Orszulak (Rochester, Minn.). One of my colleagues, Dr. David Cook, a cardiac anesthesiologist at our institution, has performed both laboratory and prospective clinical research in which I have participated. We have embraced normothermic bypass for many of our clinical procedures and research projects and have also found that there was no statistical difference between hypothermic and normothermic perfusion when analyzing for neurologic function; however, our exclusions included patients with carotid bruits and diabetes, which I believe the authors' group included.

The authors have presented timely information on a hot topic, which may offer a possible strategic compromise or a shift in the practice between hypothermia and normothermia with a tepid alternative. This may be a legitimate recommendation for the broad-based clinical cardiac surgical practice; however, I am not sure the data presented here provide irrefutable support for the tepid perfusion temperature.

I have several questions. In the manuscript the authors state that the patients were computer randomized; however, the groups were quite unequal at 37,43 , and 50 and they may be too small for heavy statistical weighting. Please explain this distribution and comment on the possibility that larger cohorts of patients may demonstrate a statistical difference.

Second, neurologic findings that prompted a CT scan were statistically different between the cold and tepid groups at $18 \%$ and $2 \%$. Could this be a reflection again of sample size or, as mentioned, related to rewarming and overheating of the brain as in the documented findings that we found in our series?

Third, the Mathews scale was used, which, as described in Lancet in 1972, used glycerol as a prospective protective agent for patients with stroke. In this study the modified version was used with nimodipine, which may not be the best method of determining and yet comparing the presence or extent of neurologic events more subtle than a stroke. I would like to ask why more universal or perhaps comparable scales, such as the National Institutes of Health or Barthel scales, were not used in this series. Could there have been more subtle findings of neurologic dysfunction not found because of the method of evaluation or sample size?

Finally, I found that the fibrinolytic study, although interesting, appears to have begun late in the series and has groups perhaps too small to evaluate. Having one group size of six patients for five variables might stretch statistical power.

I believe the authors have opened the discussion for a strategic compromise of perfusion temperature but I believe that more information is needed to finalize a recommendation.

Dr. Engelman. I was also concerned and broached the subject of group distribution with our eminent statistician, who indicated that the distribution of groups was set up in such a fashion that on the basis of a series of 300 , which was proposed, they would be essentially nearly equal. We are presenting our data on 130 patients, which clearly is less than even half of the proposed group, and that is why the groups are potentially unequal; but they are indeed random and our statistician said this was a valid presentation.

With regard to the distribution of the neurologic dysfunction, I can only say that this is what appeared. Whether it is a result of the different sample sizes between the warm and tepid groups that skewed the results, I cannot speak to, but as I mentioned, we believed the sample size was adequate.

With regard to the Mathews scale, this is our neurologist's determination of an approach that he and his associates could perform reasonably expeditiously and, with the modification as described in the manuscript, he believed that this is an approach to evaluating neurologic function that is both reliable and reproducible. The conclusions that we have reached in this study are based on details of neurologic function that we believe are appropriate.

With regard to the power of the fibrinolytic study, I would agree that clearly the number of patients at only 25 is small, but it was interesting and quite statistically significant that the difference between cold, tepid, and warm was so great in regard to at least plasminogen and prekallikrein. 